Revista Destaques Acadêmicos, Lajeado, v. 8, n. 3, 2016. ISSN 2176-3070

DOI: http://dx.doi.org/10.22410/issn.2176-3070.v8i3a2016.1055

www.univates.br/revistas

\title{
AVALIAÇÃO DO RISCO À SAÚDE EM HOMENS POR MEIO DE DIFERENTES PROTOCOLOS ANTROPOMÉTRICOS
}

\author{
Geovani André Petter ${ }^{1}$, Caito Kunrath ${ }^{2}$, Jéssica Luana Dornelles da Costa ${ }^{3}$, \\ Caroline Pieta Dias ${ }^{4}$, Carlos Leandro Tiggemann ${ }^{5}$
}

\begin{abstract}
Resumo: O objetivo do presente estudo foi avaliar o risco à saúde em homens por meio de diferentes protocolos antropométricos. Foram aferidas treze medidas antropométricas para contemplar os dez protocolos utilizados, sendo utilizadas as equações correspondentes para avaliar o nível de obesidade. Os resultados indicaram uma grande variação na classificação dos sujeitos entre os protocolos (entre 3,2 e 64,5\%), sendo os protocolos de índice de massa corporal, perímetro da cintura e os percentuais de gordura estimados pelas equações de Faulkner (1968), Pollock et al. (1976) e Pollock et al. (1980) os que descriminaram significativamente menor quantidade de sujeitos (entre 3,2 e 32,3\%). Assim, podemos concluir que existe uma variabilidade grande entre os protocolos disponíveis para classificação em risco à saúde de homens.
\end{abstract}

Palavras-chave: Antropometria. Protocolo de avaliação. Riscos à saúde.

\section{INTRODUÇÃO}

A obesidade, segundo dados da Organização Mundial da Saúde (2012), mais do que dobrou em todo o mundo desde 1980, sendo classificada como o quinto fator principal de morte. Ainda, a obesidade tem sido considerada um importante problema de saúde pública, assumindo-se como uma epidemia global. Em relação à saúde, sabe-se que o acúmulo excessivo de gordura corporal na região abdominal é um fator para o desenvolvimento de diversas patologias, aumentando o risco de se desenvolver doença arterial coronariana, hipertensão arterial, diabetes tipo II, doença pulmonar obstrutiva crônica, osteoartrite e

1 Graduado em Educação Física Bacharelado pelo Centro Universitário UNIVATES.

2 Mestrando em Educação Física pela Universidade Federal de Viçosa.

3 Graduada em Educação Física Licenciatura pelo Centro Universitário UNIVATES.

4 Doutora em Ciência do Movimento Humano.

5 Doutor em Ciência do Movimento Humano. 
certos tipos de câncer (HEYWARD; STOLARCZYK, 2000). Quanto ao controle da obesidade, deve ser priorizada a mudança comportamental baseada na dietoterapia associada à prática regular de atividade física (GUEDES; GUEDES, 2003).

Se tratando no diagnóstico da obesidade, a preocupação com índices de adiposidade corporal vem aumentando de maneira exponencial nos estudos epidemiológicos, permitindo assim direcionar as políticas públicas de manejo dessa doença. Neste sentido, diferentes metodologias de identificação da adiposidade são conhecidas, distinguindo-se entre os métodos diretos, indiretos e duplamente indiretos. Como métodos de melhor avaliação da gordura corporal a absortometria de raios $\mathrm{X}$ de dupla energia (DEXA), considerada um método indireto e padrão ouro de validação da composição corporal (COSTA, 2001). Já em relação aos métodos duplamente indiretos para identificação da obesidade, temos o Índice de massa corporal (IMC), o índice de cintura/ quadril, índice de cintura/estatura, perímetro da cintura (PC) e dobras cutâneas para equações de densidade corporal e assim estimar o percentual de gordura (\%G). Estes métodos antropométricos são considerados duplamente indiretos, porém são de baixo custo operacional, fácil aplicação e validados através do padrão ouro, o que possibilita aplicarmos em grandes amostras populacionais (COSTA, 2001).

Estudos realizados por Rezende et al. (2006, 2010), mostram que homens que apresentam massa corporal aumentada e perímetro da cintura maior ou igual a $102 \mathrm{~cm}$, têm maior probabilidade de apresentar problemas cardiovasculares em idade prematura. Além disso, Martins e Marinho (2003) mostram que indivíduos que apresentam deposição de gordura na região abdominal têm maior fator de risco para doenças cardiovasculares.

Entretanto, Gus et al. (1998) constatam que além do IMC, o perímetro da cintura e o índice cintura/quadril também podem ser utilizados como preditores de risco à saúde. Martins e Marinho (2003) afirmam que a medida de perímetro da cintura está mais associada a doenças cardiovasculares do que o risco cintura/quadril. Ainda, Kooy et al. (1993) constataram que nos homens obesos, o perímetro da cintura e o risco cintura/quadril (RCQ) se associaram à gordura visceral, o que evidencia o risco à saúde.

Além destes estudos, também Glaner, Pelegrini e Nascimento (2011) avaliaram um grupo de indivíduos heterogêneos do ponto de vista de IMC e perímetro da cintura, e constataram que os indivíduos que apresentavam IMC $\geq 25 \mathrm{~kg} / \mathrm{m}^{2}$ e perímetro cintura $>92 \mathrm{~cm}$ tinham valores de LDL-colesterol e triglicerídeos aumentados e os indivíduos que apresentaram percentual de gordura $\geq 19 \%$, encontrou-se maior proporção de sujeitos com LDL-colesterol total e triglicerídeo aumentado, demonstrando que ambas as ferramentas antropométricas utilizadas apresentavam correlação com fatores bioquímicos de risco a doenças. Porém, do ponto de vista das recomendações a respeito do ponto de corte para risco aumentado com relação ao percentual de 
gordura, os valores aparecem como sendo maiores que $25 \%$ da massa corporal (GUEDES; GUEDES, 2003), o que torna a faixa de risco à saúde mais larga quando comparado aos estudos de Glaner, Pelegrini e Nascimento (2011). Se considerarmos os estudos mais recentes, podemos questionar os pontos de corte e o verdadeiro risco quanto à adiposidade subcutânea, e quem sabe seja necessário a readequação dos valores usados para identificar o verdadeiro risco à saúde, proporcionalizado pela gordura corporal.

Dessa forma, fazendo a revisão de literatura podemos observar que existem muitas diferenças entre os estudos e as conclusões dos mesmos no que diz respeito às ferramentas que se propõem a medir a adiposidade corporal e o risco que essa por sua vez traz à saúde humana, nos remetendo ao seguinte objetivo do estudo: avaliação do risco à saúde em homens por meio de diferentes protocolos antropométricos.

\section{MÉTODOS}

O estudo caracterizou-se como de corte transversal, quantitativo e descritivo. A amostra foi composta por 31 homens que praticam atividade física duas ou mais vezes semanais, voluntários, com idade entre 35 e 50 anos, da cidade de Estrela (RS).

Como critérios de inclusão, enquadraram-se os sujeitos que apresentavam IMC entre o padrão normal $\geq 18,5 \mathrm{~kg} / \mathrm{m}^{2}$ e obesidade I $<35$ $\mathrm{kg} / \mathrm{m}^{2}$, e aparentemente saudáveis. Todos participantes assinaram o termo de consentimento livre e esclarecido, detalhando os procedimentos que foram realizados e garantindo total anonimato, sendo o projeto aprovado pelo Comitê de Ética em Pesquisa do Círculo Operário Caxiense sob o número 199.743/2013.

\section{Procedimentos para coleta}

Os voluntários da pesquisa foram informados previamente sobre o propósito da mesma e orientados para evitar exercício físico $24 \mathrm{~h}$ antes à aplicação da coleta de dados, realizada em dia único. Foram aferidas treze medidas antropométricas para contemplar os protocolos utilizados: massa corporal, estatura, quatro perímetros (braço e antebraço direito, cintura e quadril) e sete dobras cutâneas (DOC): Tricipital, Peitoral, Subescapular, Suprailíaca, Abdominal, Coxa e Perna Medial. Todas as mensurações seguiram os procedimentos sugeridos por Lopes e Ribeiro (2014).

Para determinação da massa corporal e estatura dos indivíduos foi utilizada uma balança Urano (resolução de $0,1 \mathrm{~kg}$ ) e um estadiômetro acoplado (resolução de $0,5 \mathrm{~cm}$ ), respectivamente. Indivíduos foram avaliados descalços, com os pés unidos, utilizando vestimenta leve, olhando num ponto fixo horizontal.

As medidas de perímetro foram aferidas com fita antropométrica Cescorf (resolução de $0,1 \mathrm{~cm}$ ). O perímetro da cintura foi realizado no ponto 
médio entre o último arco costal e a crista ilíaca, o perímetro do quadril obtido no ponto de maior protuberância dos glúteos, o perímetro do antebraço no ponto de maior volume do segmento estando o mesmo em posição supina, e o perímetro do braço no ponto médio entre margem lateral do acrômio e a margem superior da cabeça do rádio.

As medidas de DOC foram obtidas com a utilização de um compasso Cescorf científico (resolução de $0,1 \mathrm{~mm}$ ), e mensurada três vezes de forma não consecutiva, sendo utilizado o valor da mediana a fim de eliminar os extremos. O local de avaliação das sete dobras cutâneas avaliadas são os seguintes: DOC tricipital: realizada na face posterior do braço, paralelamente ao eixo longitudinal, no ponto médio entre margem lateral do acrômio e a margem superior da cabeça do rádio; DOC peitoral: é uma medida oblíqua em relação ao eixo longitudinal, na metade da distância entre a linha axilar e o mamilo; DOC subescapular: é executada obliquamente em relação ao eixo longitudinal, seguindo a orientação dos arcos costais, sendo localizada a dois centímetros do ângulo inferior da escápula; DOC supra-ilíaca: é obtida obliquamente em relação ao eixo longitudinal, na metade da distância entre o último arco costal e a crista ilíaca, sobre a linha axilar média; DOC abdominal: é medida, aproximadamente, a dois centímetros à direita da cicatriz umbilical, paralelamente ao eixo longitudinal; DOC coxa: é medida paralelamente ao eixo longitudinal, sobre o músculo reto femoral, na metade da distância do ligamento inguinal e da borda superior da patela; DOC perna medial: aferida no ponto de maior perímetro da perna, com o polegar da mão apoiado na borda medial da tíbia, estando o joelho em $90^{\circ}$, tornozelo em posição anatômica e o pé apoiado.

\section{Tratamento dos dados e procedimentos estatísticos}

A partir das medidas realizadas, diferentes equações foram aplicadas para identificação dos diferentes índices adotados, conforme apresentado no quadro 1. Nos protocolos de IMC, RCEst, e RCQ, as medidas avaliadas foram aplicadas em equações específicas, gerando índices individuais. Para o PC foi utilizada a própria medida para sua análise. Nos protocolos de McArdle, Katch e Katch (1981) e Faulkner (1968), as medidas coletadas e aplicadas às equações específicas, geram diretamente o valor do percentual de gordura $(\% \mathrm{G})$. Nos demais protocolos de cálculo de $\% \mathrm{G}$, as medidas avaliadas foram aplicadas nas equações específicas, permitindo alcançar valores de densidade corporal, sendo a conversão para \%G, realizada através da fórmula de regressão de Siri apud Lopes e Ribeiro (2014), expressada na equação: \%G $=[(4,95 / D C)-4,5]$ x100.

Para discriminar os sujeitos quanto ao risco à saúde, cada protocolo utilizou pontos de cortes específicos ao seu índice. Os pontos de corte foram utilizados de acordo como as referências específicas, sendo que os sujeitos foram classificados em grupo de risco ou sem risco (quadro 1), para posterior relativização (\%). 
Para o tratamento estatístico, foram utilizados valores de média e desvio padrão para descrição das variáveis. A distribuição de frequência foi utilizada para apresentar os resultados quanto ao percentual de sujeitos agrupados em risco, sendo as comparações realizadas por meio do teste Qui-Quadrado, sendo adotado um nível de significância de $\alpha \leq 0,05$, no pacote estatístico SPSS (Statistical Package for Social Science) v.21.0.

Quadro 1 - Protocolos adotados, equações específicas e pontos de corte para classificação dos sujeitos com e sem risco à saúde

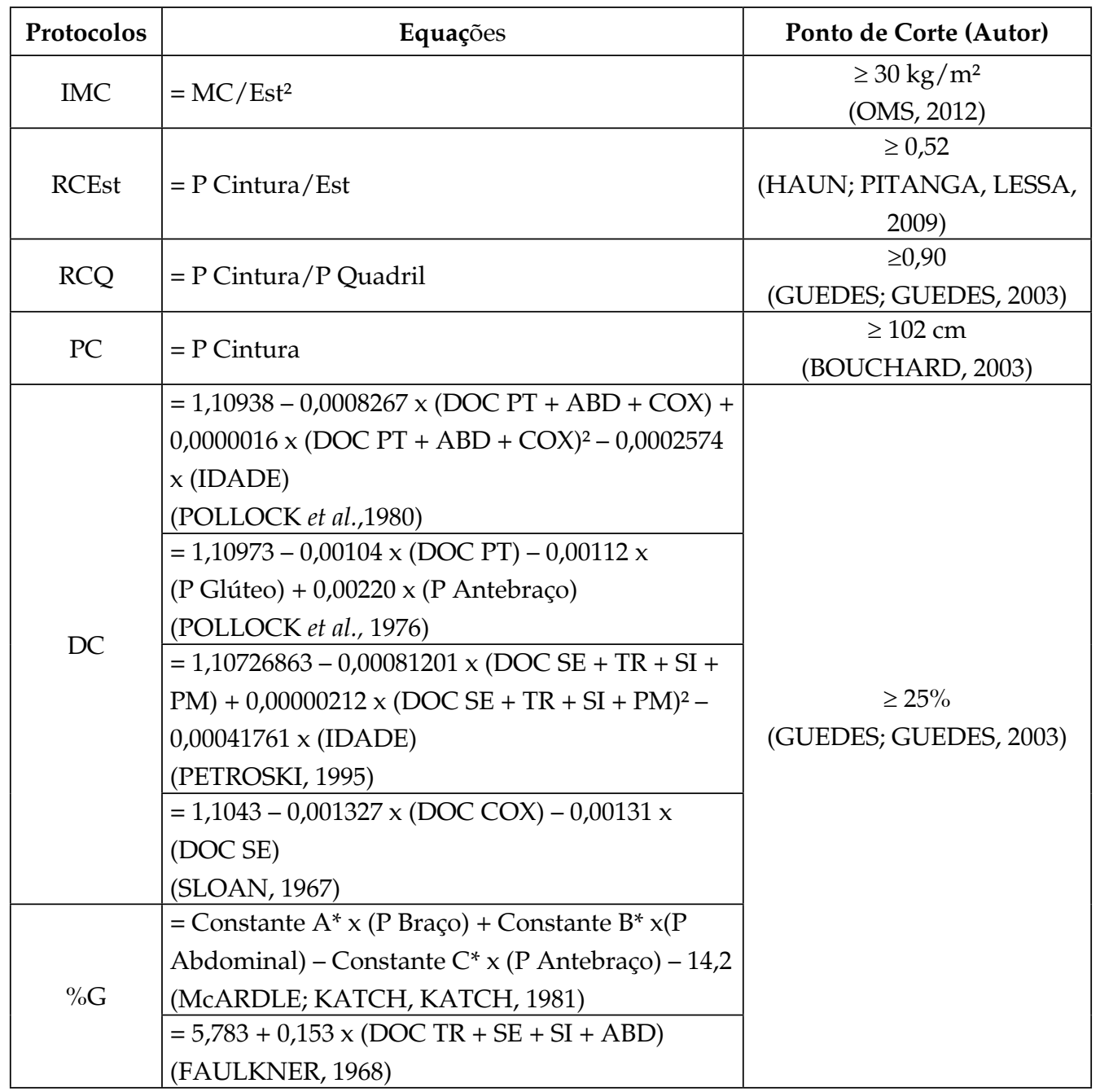

Legenda: $\mathrm{IMC}$ = índice de massa corporal; RCEst = razão cintura/estatura; RCQ = razão cintura / quadril; $\mathrm{PC}=$ perímetro da cintura; $\mathrm{DC}=$ densidade corporal; $\% \mathrm{G}=$ percentual de gordura; $\mathrm{MC}$ = massa corporal; Est = estatura; $\mathrm{P}=$ perímetro; $\mathrm{DOC}=$ dobra cutânea; $\mathrm{PT}=$ peitoral; $\mathrm{ABD}=$ abdominal $; \mathrm{COX}=$ coxa; $\mathrm{SE}=$ supra espinhal; $\mathrm{TR}=$ tricipital $; \mathrm{SI}=$ supra ilíaca; $\mathrm{PM}=$ perna medial. * Constantes de conversão de Farinatti e Monteiro (1999). 


\section{RESULTADOS}

Na tabela 1 estão apresentados os valores das variáveis mensuradas e indicadores calculados da amostra do estudo.

Tabela 1 - Valores de média, desvio padrão (DP), mínimo e máximo das variáveis e indicadores antropométricos

\begin{tabular}{|c|c|c|}
\hline Variável/Indicador & Média $\pm D P$ & Mínimo-Máximo \\
\hline Massa Corporal (kg) & $81,05 \pm 11,01$ & $63,00-111,10$ \\
\hline Estatura (m) & $1,75 \pm 0,07$ & $1,66-1,96$ \\
\hline Perímetro da Cintura (cm) & $88,50 \pm 7,52$ & $78,00-107,00$ \\
\hline Perímetro do Quadril (cm) & $99,00 \pm 18,46$ & $91,00-110,00$ \\
\hline Perímetro doBraço $(\mathrm{cm})$ & $33,00 \pm 2,48$ & $29,00-38,50$ \\
\hline Perímetro doAntebraço (cm) & $28,50 \pm 1,67$ & $25,00-32,00$ \\
\hline DOC Tricipital (mm) & $21,47 \pm 5,19$ & $10,70-33,87$ \\
\hline DOC Peitoral (mm) & $13,80 \pm 5,95$ & $6,93-29,30$ \\
\hline DOC Subescapular (mm) & $25,73 \pm 7,47$ & $9,67-39,57$ \\
\hline DOC Supra-ilíaca (mm) & $24,23 \pm 8,54$ & $8,67-39,40$ \\
\hline DOC Abdominal (mm) & $36,67 \pm 10,08$ & $13,30-50,03$ \\
\hline DOC Coxa (mm) & $17,23 \pm 6,48$ & $7,90-37,30$ \\
\hline DOC Perna Medial (mm) & $9,87 \pm 4,12$ & $4,53-23,07$ \\
\hline $\mathrm{IMC}\left(\mathrm{kg} / \mathrm{m}^{2}\right)$ & $26,54 \pm 2,82$ & $20,19-31,74$ \\
\hline RCEst & $0,51 \pm 0,04$ & $0,44-0,59$ \\
\hline RCQ & $0,89 \pm 0,04$ & $0,81-0,99$ \\
\hline \%G Faulkner (1968) & $22,27 \pm 4,16$ & $12,67-28,14$ \\
\hline \%G McArdle, Katch e Katch (1981) & $23,14 \pm 5,31$ & $12,17-32,63$ \\
\hline \%G Pollock et al. (1976) & $17,19 \pm 4,76$ & $8,96-25,00$ \\
\hline \%G Pollock et al. (1980) & $21,41 \pm 5,39$ & $10,08-29,93$ \\
\hline \%G Petroski (1995) & $26,85 \pm 4,81$ & $15,80-34,37$ \\
\hline \%G Sloan (1967) & $21,67 \pm 7,31$ & $10,06-39,28$ \\
\hline
\end{tabular}

Legenda: $\mathrm{DOC}=$ dobra cutânea; IMC = índice de massa corporal; RCEst = razão cintura/ estatura; $\mathrm{RCQ}=$ razão cintura $/$ quadril; $\% \mathrm{G}=$ percentual de gordura.

Os resultados do estudo indicam que a quantidade percentual de sujeitos classificados em risco à saúde entre os 10 protocolos utilizados variaram entre 3,2 e 64,5\% (FIGURA 1). Os protocolos de IMC, PC, \%G Faulkner (1968), \%G Pollock et al. (1976) e Pollock, Schmidt e Jackson (1980) descriminaram 
significativamente uma menor quantidade de sujeitos com risco de saúde (entre 3,2 e 32,3\%) quando comparado aos protocolos de RCEst, RCQ, \%G McArdle, Katch e Katch (1981), \%G Petrosky (1995) e \%G Sloan (1967) (entre 38,7 e 64,5\%) $(\mathrm{p}<0,05)$.

Figura 1 - Quantidade relativa de sujeitos (\%) apresentando risco relativo à saúde, conforme os diferentes protocolos adotados



Legenda: IMC = índice de massa corporal; RCEst = razão cintura/estatura; RCQ = razão cintura / quadril; $\mathrm{PC}=$ perímetro da cintura; $\% \mathrm{G}=$ percentual de gordura. ${ }^{*}$ diferença significativa $\mathrm{p}<0,05$.

\section{DISCUSSÃO}

Devido aos inúmeros riscos à saúde que estão associados com a obesidade, a mesma vem sendo definida como uma doença crônica não transmissível, tornando-se um problema de saúde pública afetando todas as faixas etárias e grupos socioeconômicos (HAUN; PITANGA; LESSA, 2009). O diagnóstico correto da obesidade ou o sobrepeso requer que os níveis de risco sejam identificados e isto, frequentemente, necessitando de formas adequadas de quantificação. A OMS (2004) tem indicado o uso de variáveis antropométricas para o monitoramento dos fatores de risco das doenças crônicas não transmissíveis.

No presente estudo, alguns protocolos se assemelharam quanto aos indivíduos considerados com risco à saúde, entre eles: O RCEst, o RCQ 
e os protocolos de McArdle (1981), Pollock et al. (1980) e Sloan (1967), os quais indicaram de 32,3 a 45,2\% dos indivíduos em risco à saúde. Diversos estudos têm demonstrado que a RCEst é um bom discriminador de obesidade abdominal relacionada a fatores de risco cardiovascular, bem como de risco coronariano elevado (PITANGA; LESSA, 2006). Diferentemente do proposto pela OMS (2004), este estudo sugere que o PC não foi o melhor preditor de risco à saúde.

Em estudo de Fogaça et al. (2014) observou-se que em indivíduos cardiopatas a RCQ e o PC apresentam-se acima dos valores desejados. Levando em consideração que a RCQ e o RCEst utilizam a medida do perímetro da cintura, pode-se sugerir que isso torna maior a chance de se assemelharem quanto ao resultado, indo ao encontro do resultado encontrado no estudo de Haun, Pitanga e Lessa (2009). Por outro lado, conforme Balkau et al., (2007) o PC seja uma medida diretamente relacionada à quantidade de tecido adiposo intraabdominal e a mesma tenha sido associada a fatores de risco cardiovascular, no presente estudo foram encontrados poucos indivíduos com risco à saúde relacionados à esta medida.

No estudo de Chagas et al. (2011) com indivíduos submetidos à cineangiocoronario-grafia por suspeita de doença arterial crônica, verificouse que nenhum dos parâmetros antropométricos estudados foi fator de risco independente para a carga aterosclerótica coronariana. Em relação ao IMC, os resultados corroboram os achados de estudos prévios, em que esse parâmetro não está associado à aterosclerose significativa (AUER et al., 2005; HODGSON et al., 1994), sendo que uma das limitações do IMC é a não diferenciação entre diferentes composições corporais. Por outro lado, a capacidade da RCQ em se correlacionar com risco cardiovascular pode estar ligada ao fato dessa variável contemplar dois aspectos diferentes como: (1) a variação da gordura subcutânea e visceral abdominal mensurada pela circunferência da cintura e (2) a estrutura pélvica, músculo glúteo e gordura subcutânea glútea mensurada pela circunferência do quadril, ou seja, anatomicamente, a RCQ pode indicar melhor distribuição da gordura corporal (SEIDELL et al., 2001).

Além disso, no estudo de Chagas et al. (2011) os resultados apontaram a inexistência de associação de RCEst com carga aterosclerótica de encontro do nosso estudo, onde esta medida apontou um maior número de indivíduos com risco à saúde. Neste sentido, a RCEst tem sido fortemente correlacionada com gordura intra-abdominal e a maior risco para doença cardiovascular (ASHWELL; COLE; DIXON, 1996; SCHNEIDER et al., 2007).

Foi verificado que houve protocolos que discriminaram poucos indivíduos com risco à saúde, entre eles: o IMC, PC, e \%G segundo Pollock et al. (1976) e Faulkner (1968) e este fator faz a discussão ainda maior sobre quais protocolos são mais rigorosos ou não, perante os indivíduos do presente estudo. Entretanto, o protocolo de Petroski (1995) mostrou-se rigoroso, indicando mais de $60 \%$ dos homens como a saúde em risco. A amostra do estudo de Petroski 
(1995) foi composta por indivíduos com as mesmas características do presente estudo e sua equação considera o fator idade do indivíduo, o que leva a acreditar que é confiável em relação aos resultados deste estudo.

Em relação à comparação entre os diferentes protocolos, diferentes estudos têm se proposto a esta investigação. Rios e Barros (2010) avaliaram 30 homens e 30 mulheres universitários comparando o protocolo de dobras cutâneas com o de bioimpedância. Os resultados indicaram semelhantes valores de percentual de gordura entre os homens $(15,34 \pm 5,06 \%$ e $15,98 \pm 5,36 \%)$, contudo, diferentes valores entre as mulheres $(23,86 \pm 4,75 \%$ e $21,89 \pm 6,37 \%)$.

Nunes et al. (2009) avaliaram a confiabilidade da classificação do estado nutricional obtida através do IMC e três diferentes métodos de composição corporal (dobras cutâneas, bioimpedância bipolar e tetrapolar) em indivíduos diabéticos tipo 1 e não diabéticos. Quando analisados os resultados do grupo controle (saudáveis), verificou-se que o protocolo de dobras cutâneas teve concordância com o IMC em $75,6 \%$ da classificação dos sujeitos, $62,1 \%$ no protocolo de bioimpedância bipolar e $59,4 \%$ no protocolo de bioimpedância tetrapolar. Em outro estudo, Yao et al. (2002) identificaram que mais de $30 \%$ dos indivíduos (homens e mulheres com idades entre 35 e 49 anos) que apresentaram excesso de gordura corporal foram classificados como eutróficos pelo IMC, demonstrando sua baixa sensibilidade na identificação do excesso de gordura corporal.

Importante acrescentar que o perfil antropométrico dos indivíduos varia de região para região, assim como os resultados de uma equação e a sua validade (COSTA, 2001). Dessa forma, os resultados encontrados podem variar de acordo com a região avaliada assim como fatores relacionados à etnia, sexo, idade ou nível de atividade física. Mas, além das especificidades de cada protocolo, um aspecto que ainda parece ser dúbio e carente de extensa investigação, diz respeito aos pontos de cortes adotados para cada protocolo.

Rezende et al. (2007) apresenta em seu artigo de revisão aspectos de grande relevância para o tema. Um deles diz respeito à dificuldade de validar os pontos de cortes originários de amostras específicas para sujeitos com perfis diferenciados. Além disso, a utilização de pontos de cortes arbitrários faz com que sejam estabelecidas estimativas errôneas da composição corporal e consequente classificação. Outra consideração importante diz respeito à baixa quantidade de equações realmente validadas por meio de padrão ouro (pesagem hidrostática e a absorção do raio $X$ de dupla energia - DEXA), além de não descriminar os sujeitos quanto a sua etnia e nível de atividade física, utilizando muitas vezes amostras muito específicas (militares e universitários) ou pequenas.

Considerando-se que o excesso de peso corporal está associado ao aparecimento de doenças cardiovasculares e mortalidade, alguns indicadores antropométricos têm se destacado pela sua simplicidade operacional e boa acurácia permitindo a deteç̧ão de indivíduos com risco à saúde, possibilitando 
o mapeamento de grupos populacionais frente a esses riscos. Contudo, uma consideração muito importante a ser destacada é o entendimento que os pontos de cortes são advindos de um modelo estatístico.

A exemplo disso, a OMS (2012) utilizou em relação ao IMC os percentis $\geq 85$ e $\geq 95$ para classificar os sujeitos adultos com sobrepeso e obesidade, respectivamente. Este procedimento é adotado pelos demais protocolos, contudo utilizando muitas vezes outros perfis de amostra, dificultando o entendimento de como os protocolos se relacionam. Relacionar os diferentes protocolos entre si, visando identificar a associação entre os mesmos é de considerável importância, mas ainda mais importante, é a necessidade de estudos de base populacional utilização diferentes protocolos associados aos diferentes desfechos (doenças cardiovasculares, diabetes, outras), estratificados por fatores muitas vezes não considerados (etnia, nível de atividade física, outros).

\section{CONCLUSÃO}

Como conclusão de nosso estudo, verificamos que a quantidade percentual dos homens classificados em risco à saúde entre os 10 protocolos utilizados variou entre 3,2 e $64,5 \%$. Embora os protocolos utilizados tenham respeitado aspectos quanto ao sexo e a faixa etária, não foram observados outros fatores, como por exemplo, a etnia e o perfil da amostra em que o protocolo foi originário. Desta forma, parece ser sensato sugerir mais do que um protocolo de avaliação antropométrica para indicar um marcador com possíveis riscos à saúde.

\section{REFERÊNCIAS}

ASHWELL, Margaret; COLE, Timothy J.; DIXON, Adrian K. Ratio of waist circumference to height is strong predictor of intra-abdominal fat. British Medical Journal, v. 313, n. 56, p. 559-60, 1996.

AUER, Johann W. et al. Obesity, body fat and coronary atherosclerosis. International Journal of Cardiology, v. 98, n. 2, p. 227-35, 2005.

BALKAU, Beverley et al. International Day for the Evaluation of Abdominal Obesity (IDEA): a study of waist circumference, cardiovascular disease, and diabetes mellitus in 168,000 primary care patients in 63 countries. Circulation, v.116, n.17, p.1942-51, 2007.

BOUCHARD, Claude. Atividade física e obesidade. 1 ed. São Paulo: Manole, 2003.

CHAGAS, Patrícia. et al. Associação de diferentes medidas e índices antropométricos com a carga Aterosclerótica Coronariana. Arquivo Brasileiro de Cardiologia, v. 97, n.5, p. 397-401, 2011. 
COSTA, Roberto F. da. Composição Corporal: teoria e prática da avaliação. 1 ed. São Paulo: Manole, 2001.

FAULKNER, John A. Physiology of swimming and diving. In: H. FALLS. Exercise Physioloy, Baltimore Academic Press, 1968.

FARINATTI, Paulo de T. V.; MONTEIRO, Walace D. Fisiologia e Avaliação Funcional. 3 ed. Rio de Janeiro: Sprint, 1999.

FOGAÇA, Luciano T. et al. Avaliação da razão cintura-quadril de indivíduos cardiopatas. Revista Brasileira de Ciências da Saúde, v. 40, n.12, p. 1-6, 2014.

GLANER, Maria F.; PELEGRINI, Andrea; NASCIMENTO, Thales B. R. Perímetro do abdômen é o melhor indicador antropométrico de riscos para doenças cardiovasculares. Revista Brasileira de Cineantropometria \& Desempenho Humano, v. 13, n 1, p.1-7, 2011.

GUEDES, Dartagnan P.; GUEDES, Joana E. R. P. Controle do peso corporal: composição corporal, atividade física e nutrição. 2 ed. Rio de Janeiro: Shape, 2003.

GUS, Miguel. et al. Associação entre diferentes indicadores de obesidade e prevalência de hipertensão arterial. Arquivos brasileiros de cardiologia, v. 70, n. 2, p. 111-114, 1998.

HAUN, Danilo R.; PITANGA, Francisco J. G.; LESSA, Ines. Razão cintura/estatura comparado a outros indicadores antropométricos de obesidade como preditor de risco coronariano elevado. Revista da associação médica brasileira, v. 55, n. 6, p. 705-711, 2009.

HEYWARD, Vivian H.; STOLARCZYK, Lisa M. Avaliação da composição corporal aplicada. 1 ed. São Paulo: Manole, 2000.

HODGSON, John M. et al. Coronary atherosclerosis in relation to body fatness and its distribution. International Journal of Obesity and Related Metabolic Disorders, v. 18, n. 1, p. 41-6, 1994.

KOOY, Karin V. D. et al. Abdominal diameters as indicators of visceral fat : comparison between magnetic resonance imaging and anthropometry. The British Journal of Nutrition, v. 70, n.1, p. 47-58, 1993.

LOPES, André L.; RIBEIRO, Gustavo dos S. Antropometria aplicado à saúde e ao desempenho esportivo: uma abordagem a partir da metodologia ISAK. 1. ed. Rio de Janeiro: Rubio, 2014.

MARTINS, Ignes S.; MARINHO, Sheila P. O potencial diagnóstico dos indicadores da obesidade centralizada. Revista de saúde pública, v. 37, n. 6, p. 760-767, 2003.

McARDLE, William D.; KATCH, Frank I.; KATCH, Victor L. Exercise Physiology: Energy, nutrition and human performance. Philadelphia: Lea \& Febiger, 1981.

NUNES, Raquel R. et al. Confiabilidade da classificação do estado nutricional obtida através do IMC e três diferentes métodos de percentual de gordura corporal em pacientes com diabetes melito tipo 1. Arquivos Brasileiro de Endocrinologia e Metabologia, v. 53, n. 3, p. 360-367, 2009. 
OMS, Organização Mundial da Saúde. 2012. Disponível em: <www.who.int/ research/es>. Acesso em: 24 de out. 2012.

OMS, Organização Mundial da Saúde. Obesidade: prevenindo e controlando a epidemia global. 1.ed. Roca, 2004.

PETROSKI, Edio L. Desenvolvimento e validação de equações generalizadas para estimativa da densidade corporal em adultos. 1995. E-book. Tese (doutorado em ciências do movimento humano) - Universidade Federal de Santa Maria, Santa Maria. 1995.

PITANGA, Francisco J. G.; LESSA, Ines. Razão cintura-estatura como discriminador do risco coronariano de adultos. Revista da Associação Médica Brasileira, v. 52, n. 3, p. 157-61, 2006.

POLLOCK, Michael L. et al. Measurement of cardiorespiratory fitness and body composition in the clinical setting. Comprehensive Therapy, v. 6, n. 3, p. 12-27, 1980.

POLLOCK, Michael L. et al. Prediction of body density in Young and middle-aged men. Journal of Applied Physiology, v. 30, n. 3, p. 300-304, 1976.

REZENDE, Fabiane A. C. et al. Aplicabilidade do índice de massa corporal na avaliação da gordura corporal. Revista Brasileira de medicina do esporte, v. 16, n. 2, p. 90-94, 2010.

REZENDE, Fabiane A. C. et al. Índice de massa corporal e circunferência abdominal: associação com fatores de risco cardiovascular. Arquivos brasileiros de cardiologia, v. 87, n. 6, p. 666-671, 2006.

REZENDE, Fabiane. et al. Revisão crítica dos métodos disponíveis para avaliar a composição corporal em grandes estudos populacionais e clínicos. Archivos Latinoamericanos De Nutricion, v. 57, n. 4. p. 327-334, 2007.

RIOS, Daniela G.; BARROS, Cristiano L. Comparação de diferentes métodos de estimativa do percentual de gordura em estudantes universitários. Revista Mineira de Ciências da Saúde, v. 1, n. 2, p. 21-27, 2010.

SCHNEIDER, Harald J. et al. Accuracy of anthropometric indicators of obesity to predict cardiovascular risk. The Journal of Clinical Endocrinology and Metabolism, v. 92, n. 2, p. 589-94, 2007.

SEIDELL, Jacob C. et al. Waist and hip circumferences have independent and opposite effects on cardiovascular disease risk factors: the Quebec Family Study. The American Journal Clinical Nutrition, v. 74, n. 3, p. 315-21, 2001.

SLOAN, Angela. Estimation of body fat in young men. Journal of Applied Physiology, v. 23, n. 3, p. 311-315, 1967.

YAO, Manjiang. et al. Field Methods for Body Composition Assessment Are Valid in Healthy Chinese Adults. The Journal of Nutrition, v. 132, n. 02, p. 310-317, 2002. 